\title{
Ampullary region tumors: A case of an intra-ampullary papillary-tubular neoplasm (IAPN)
}

\author{
Georg Hagleitner ${ }^{1 *}$ and Franz A Fellner ${ }^{1,2}$ \\ ${ }^{1}$ Central Radiology Institute, Kepler University Hospital, Medical Faculty of the Johannes Kepler University, Linz, Austria \\ ${ }^{2}$ Medical Faculty of the Friedrich-Alexander-University of Erlangen-Nürnberg, Erlangen, Germany
}

\section{Background}

Intra-ampullary papillary tubular neoplasms (IAPNs) are a rare entity of neoplasms in the region of the papilla of Vateri $(0,5 \%$ of all gastrointestinal cancers) [1-7]. IAPNs are the second most common entity of periampullary cancers (PACs, 16-28\%) after pancreatic adenocarcinoma [4]. The ampullary region is a histologically and physiologically complex region where three different structures meet: the common bile duct (CBD), pancreatic duct (PD) and duodenum [1]. In the family of tumoral intraepithelial neoplasms of the pancreaticobiliary tract IAPNs form a distinct entity and should not be confused with intraductal tubulopapillary neoplasms (ITPNs) of the pancreas or the biliary tract, intraductal papillary mucinous neoplasms (IPMNs) or intracholecystic papillary tubular neoplasms (ICPNs) [1,2]. Most cases of IAPN are associated with a high-grade dysplasia or small parts of invasive carcinoma [2,3]. IAPNs typically follow the „adenomacarcinoma sequence" and can evolve to pancreatic carcinoma $[1,5]$. Because of the malign potential of IAPNs and other malign PACs it is important to early recognize and treat these lesions $[1,4,7]$. Diagnostic imaging options include sonography, computed tomography (CT), magnetic resonance imaging (MRI), endoscopic retrograde cholangiopancreatography (ERCP), and endoscopic ultrasound $[4,8]$. Definitive diagnosis is made histologically after sampling (ERCP) or resection [6,7].

\section{Case report}

We report a 56-year old male patient, who presented in our emergency department with recurrent abdominal pain in the right upper quadrant for about six weeks. No jaundice, nausea or vomiting was present. Initial laboratory findings showed elevated levels of gamma-glutamyl transferase (GGT; $313 \mathrm{U} / \mathrm{L}$ ) and slightly elevated levels of alkaline phosphatase (ALP; $131 \mathrm{U} / \mathrm{L}$ ), aspartate transaminase (AST; $58 \mathrm{U} / \mathrm{L}$ ) and alanine transaminase (ALT; $87 \mathrm{U} / \mathrm{L})$. C-reactive protein (CRP) was slightly elevated $(2,8 \mathrm{mg} / \mathrm{dl})$.

To exclude a cholecystitis, cholecystolithiasis or choledocholithiasis an ultrasound examination was performed, which showed a prepapillary solid lesion without posterior acoustic shadowing (Figure 1). No perfusion with Doppler ultrasonography was present (not shown). The intra- and extrahepatic bile duct system was dilated. No suspect lymph nodes were found.

A subsequent contrast-enhanced MRI of the pancreas with MR cholangiography confirmed intra- and extrahepatic cholestasis and a contrast enhancing mass in the papillary region with mild diffusion restrictions. The CBD showed mild signs of cholangitis. The PD showed no dilatation, the pancreatic parenchyma was inconspicuous. Along the hepatoduodenal ligament and at the liver hilum some inconspicuous lymph nodes were found. Radiological tumor staging was completed with a multi-detector $\mathrm{CT}$ of the thorax and abdomen with multi-phase imaging of the pancreas. No remote metastases were found, the findings from the MRI examination were confirmed (Figures 2-7).

Tumor marker serum carbohydrate antigen 19-9 (CA 19-9) was slightly elevated $(32,9 \mathrm{kU} / \mathrm{L})$. The tissue samples from an ERCP showed the pattern of a papillary adenoma with partially high-grade dysplasia.

Interdisciplinary tumor conference recommended surgical resection. A pancreaticoduodenectomy (Whipple's procedure) with antrectomy and lymphadenectomy was performed. Frozen section analysis showed R0 resection and normal preaortic and hepatic hilum lymph nodes.

Further histological workup was consistent with an IAPN, positive for Cytokeratin 20 and CDX2 and low positivity for EMA (MUC1), MUC2 and MUC6. No signs of cancer were found, all lymph nodes were negative for metastases. Final UICC 2017 staging was pTis N0 (0/17) R0 M0.

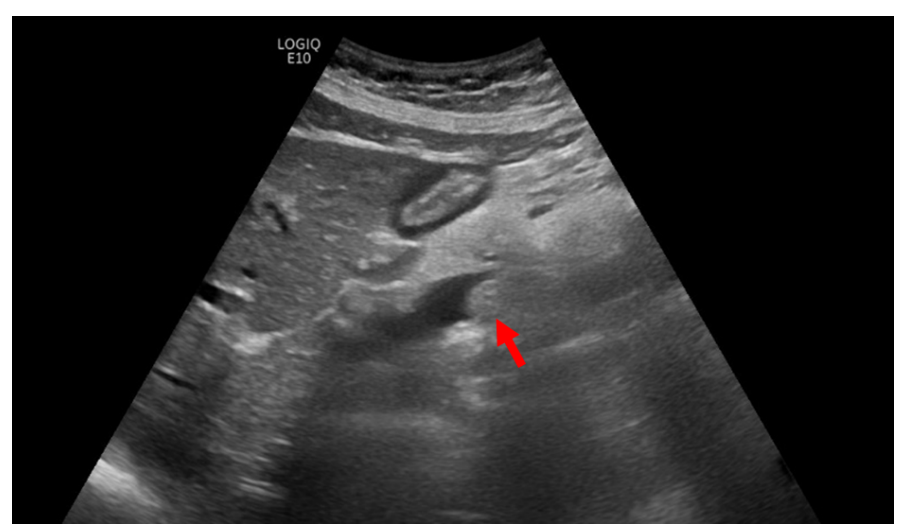

Figure 1. Ultrasonography shows a solid lesion in the ampullary region (arrow). No posterior acoustic shadowing

${ }^{*}$ Correspondence to: Georg Hagleitner, Central Radiology Institute, Kepler University Hospital, Medical Faculty of the Johannes Kepler University, Linz, Austria, E-mail: georg.hagleitner@kepleruniklinikum.at

Key words: pancreas, ampullary neoplasm, intra-ampullary papillary tubular neoplasms, IAPN, MRI

Received: May 06, 2020; Accepted: May 22, 2020; Published: May 25, 2020 


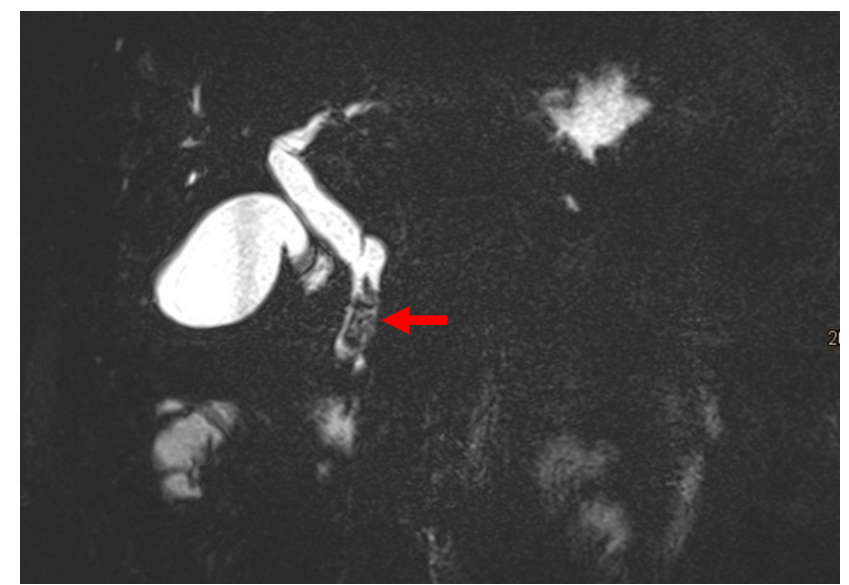

Figure 2. MRCP shows a filling defect in the ampulla (arrow) and dilatation of the CBD

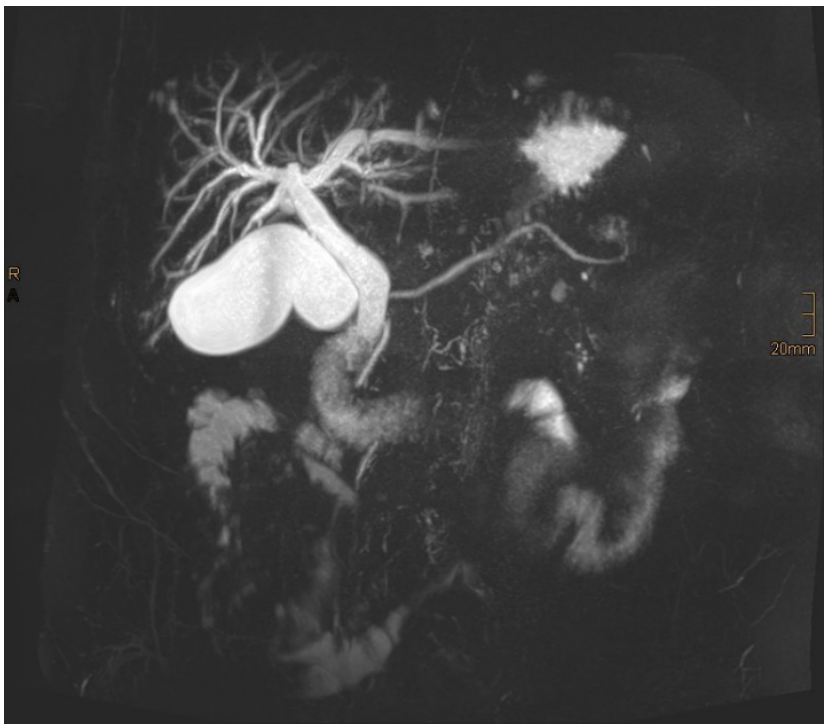

Figure 3. Maximum intensity projection (MIP) from MRCP. Intra- and extrahepatic cholestasis. No dilatation of the PD

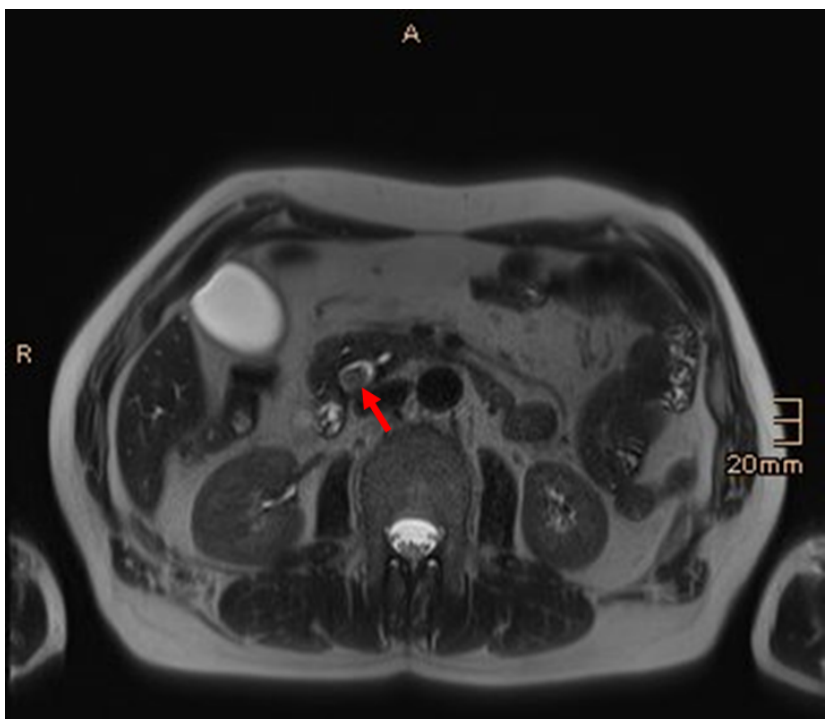

Figure 4. T2-weighted axial image demonstrates a solid lesion in the CBD (arrow)

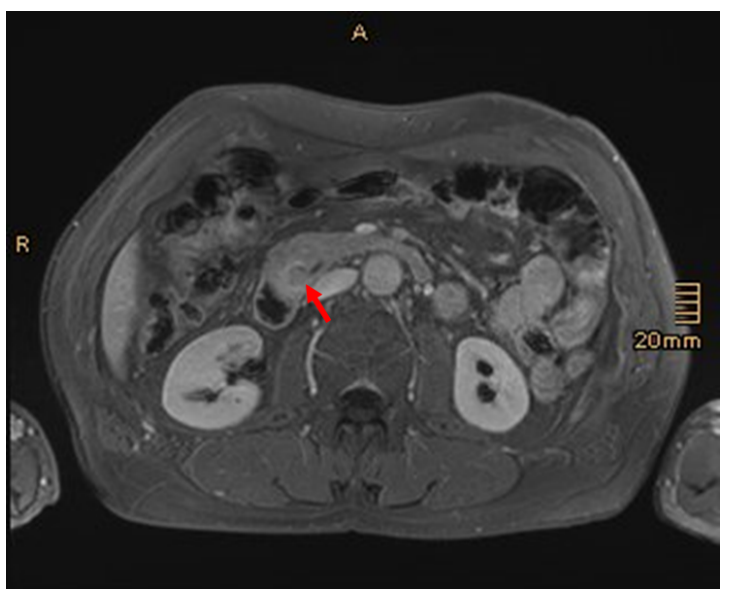

Figure 5. Gd-enhanced T1-weighted image reveals contrast enhancement of the lesion (arrow)

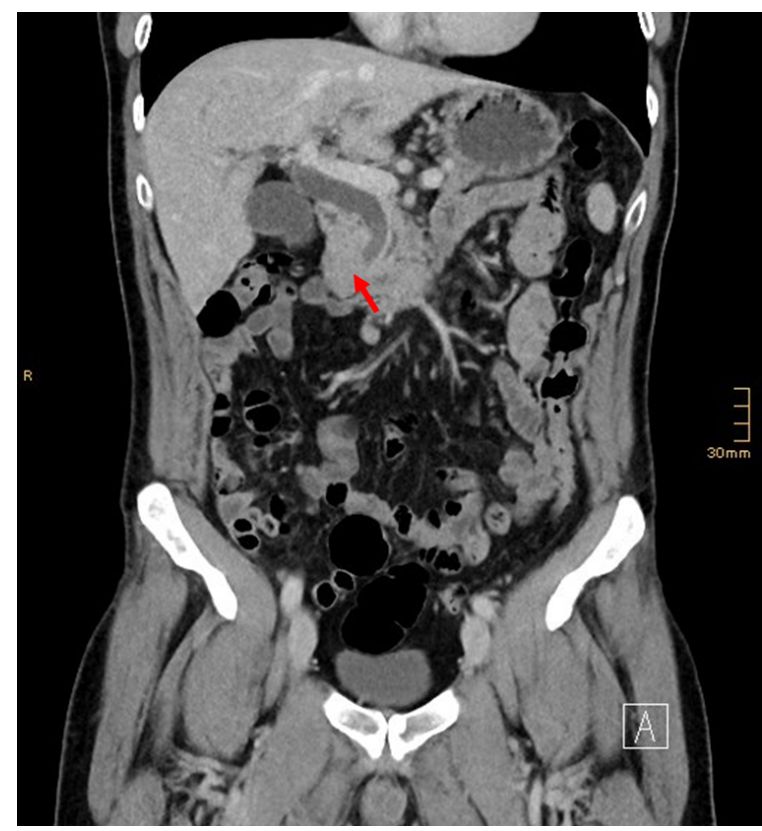

Figure 6. Contrast enhanced CT scan of the suspicious lesion (arrow)

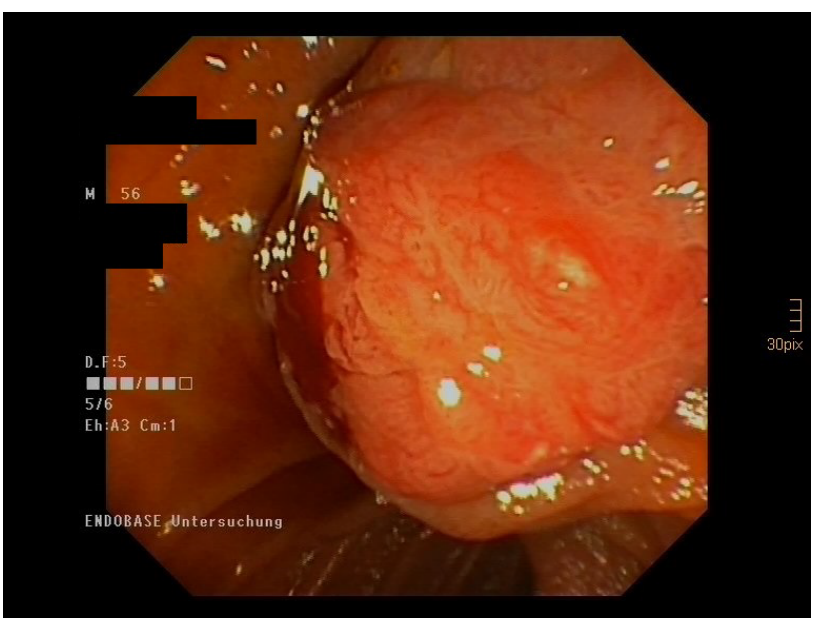

Figure 7. ERCP image of the solid tumor in the ampulla 


\section{Discussion}

Clinical presentation of patients with IAPNs is unspecific and mostly related to obstruction of the CBD and/or PD [6]. Typical symptoms are abdominal pain, jaundice, weight loss, diarrhea, diabetes, nausea and vomiting $[4,5]$. Thus, laboratory findings can be normal or show signs of cholestasis with or without cholangitis. Age peak is from the sixth to seventh decade [6]. Due to symptomatic patients in early stage IAPNs lead to early diagnosis and better prognosis compared to more distally localized pancreatic or biliary neoplasms [5].

Primary diagnostic workup through ultrasonography is usually targeted towards frequent causes of right/left upper quadrant pain to rule out cholecystolithiasis, cholecystitis, choledocholithiasis and pancreatitis.

The ampullary region is a complex anatomic region, which can be challenging for radiologists to assess [9]. About $62 \%$ of ampullary lesions manifest on CT and MR images as irregular filling defect in the ampulla [8]. For cross-sectional imaging it is recommended to acquire multiplanar images and contrast enhanced images to define tumor extension, invasion of surrounding structures and vasculature and to differentiate a solid lesions from a choledocholith $[4,8,9]$. In MRI, additional diffusion-weighted imaging increases detection of ampullary carcinoma, whilst unenhanced T1- and T2-weighted signal intensity is unspecific regarding malignancy [10]. After contrast administration, malignant lesions are usually hypoenhancing on cross-sectional images [10]. MR cholangiography is best to visualize intra- and extrahepatic cholestasis and the dilatation of the PD [8]. Besides lack of radiation, the detection and classification of liver lesions is better in MRI $[4,8]$. CT is useful in tumor staging to diagnose remote metastases and to resolve vascular details and anatomy, although IAPNs are less likely to involve the surrounding vasculature [4].

Lesions of the ampulla can be neoplastic, non-neoplastic, inflammatory or congenital [8]. Important differential diagnosis for ampullary and periampullary lesions are ampullary cancer, ampullary adenoma, distal CBD cholangiocarcinoma, duodenal adenoma, GIST, periampullary lipoma, pancreatic adenocarcinoma, PNET, IPMN (main duct type), other cystic pancreatic neoplasms, papillary stenosis, choledocholithiasis, pancreas divisum, santorinicele, choledochocele, groove pancreatitis, autoimmune pancreatitis, pancreaticoduodenal artery pseudoaneurysm, Brunner's gland hyperplasia and hamartoma, duodenitis, duodenal Crohn's disease, duodenal diverticula and duodenal perforation $[8,9]$.

ERCP can be diagnostic in visualizing the lesion and tissue sampling. In smaller, endothelial/non-invasive lesions ERCP can be therapeutic [4]. Additional information about local tumor growth, submucosal and intraductal invasion and more precise tissue sampling can be acquired through endoscopic ultrasound [4].
Adsay et al. emphasize on the differentiation of intraepithelial neoplasms of the ampullo-pancreatobiliary tract in five distinct subtypes: the IAPNs at the ampulla, the IPMNs and ITPNs along the $\mathrm{PD}$, the IPMNs of the biliary tract and the ICPNs in the gallbladder [2]. Primary IAPNs with a size larger than $3 \mathrm{~cm}$, periampullary lymph nodes larger than $2 \mathrm{~cm}$ and elevated levels of CA 19-9 greater than 200 $\mathrm{ng} / \mathrm{ml}$ are associated with a higher risk of malignancy and/or early recurrence after resection [4,7].

In most cases without infiltration or metastases, surgical resection is recommended [6]. Poley et al. promote three surgical options: Pancreaticoduodenectomy (Whipple's procedure), surgical local excision (ampullectomy) or endoscopic ampullectomy [6].

\section{Conclusion}

Lesions of the anatomically and histologically complex ampullary region show a large spectrum of differential diagnosis and can be challenging for radiologists to assess. Imaging plays an essential role in excluding benign causes of CBD obstruction and staging benign and malignant lesions for further therapy.

\section{Disclosure}

No conflicts of interest. No grants or financial supports.

\section{References}

1. Pant M, Pehlivanoglu B, Erbarut-Seven I, Adsay V (2018) Intra-ampullary papillarytubular neoplasm: an update on the ampullary counterparts of pancreatobiliary intraductal neoplasms. AJSP: Reviews \& Reports 23: 38-43.

2. Adsay V, Ohike N, Tajiri T, Kim GE, Krasinskas A, et al. (2012) Ampullary region carcinomas: Definition ans site specific classification with delineation of fou clinicopathologically and prognostically distinct subsets in an analysis of 249 cases. Am J Surg Pathol 36: 1592-1608

3. Gracient A, Delcenserie R, Chatelain D, Brazier F, Lemouel JP, et al. (2019) Endoscopic and surgical ampullary tumor: the patient populations are not the same. J Visc Surg in press.

4. Zheng-Pywell R, Reddy S (2018) Ampullary Cancer. Surg Clin N Am in press.

5. Ren D, Li D, Jin X, Meng Z, Wu H (2019) Intra-ampullary papillary-tubular neoplasm: a population-based analysis. Med Sci Monit 25: 7332-7341. [Crossref]

6. Poley JW, Campos S (2019) Methods and outcome of the endoscopic treatment of ampullary tumors. Ther Adv Gastrointest Endosc 13: 1-13. [Crossref]

7. Park HM, Park SJ, Han SS, Hong SK, Hong EK, et al. (2019) Very early recurrence following pancreatoduodenectomy in patients with ampullary cancer. Medicine 98:44(e17711). [Crossref]

8. Nikolaidis P, Hammond NA, Day K, Yaghmai V, Wood CG, et al. (2014) Imaging features of benign and malignant ampullary and periampullary lesions. RadioGraphics 34: 624-641.

9. Raman SP, Fishman EK (2013) Abnormalities oft he distal common bile duct and ampulla: diagnostic approach and differential diagnosis using multiplanar reformations and 3D imaging. AJR 203: 17-28.

10. Jang KM, Kim SH, Lee SJ, Park HJ, Choi D, et al. (2013) Added value of diffusionweighted MR imaging in the diagnosis of ampullary carcinoma. Radiology 266;2: 491501

Copyright: (C2020 Hagleitner G. This is an open-access article distributed under the terms of the Creative Commons Attribution License, which permits unrestricted use, distribution, and reproduction in any medium, provided the original author and source are credited. 